\title{
A Case Report: Ruptured Pyogenic Liver Abscess
}

\author{
Dewi Roziqo $^{1}$, Budi Laraswati ${ }^{1}$ \\ Email: dewiroziqo@gmail.com \\ ${ }^{1}$ Department of Radiology, Faculty of Medicine, Airlangga University, Surabaya, Indonesia
}

\begin{abstract}
Background: Most causative of liver abscess, in about $90 \%$ of all cases, are pyogenic abscess. One of the rare and dangerous complications of pyogenic liver abscess (PLA) that requires urgent management, even with nonspecific signs and symptoms, is abscess rupture.

Case Presentation: A 27 years old male patient came to the hospital with persistent right upper abdominal pain. The pain started one week ago after the patient fell from the stairs, where the right side of the abdomen hit the stairs. Abdominal ultrasound revealed heteroechoic lesion with ill borders, irregular margin, and internal moving echo (+) in the right lobe of the liver, which on color doppler examination showed minimal perilesional vascularization. The patient underwent abdominal computed tomography (CT) scan with contrast four days later with showed rim enhancing semisolid lesion (23-33 HU) with air density $(-925 \mathrm{HU})$ within it in, in the segments V to VIII of the right lobe of the liver partially ruptured in the periphery of segments VI-VII of the liver lobe with pneumoperitoneum and ascites. A week later, a laparotomy was performed, and the culture of the pus specimen revealed growth of Escherichia coli.

Conclusion: Based on clinical symptoms and imaging, PLA is not without any challenges in diagnosis. The key to avoiding misdiagnosis of ruptured PLA is a meticulous examination of the clinical and imaging also vigilant monitoring.
\end{abstract}

Keyword: Liver abscess, Abdominal ultrasound, Abdominal CT scan, rupture

\section{Introduction}

Liver abscess is defined as a collection of purulent material encapsulated in the liver parenchyma accompanied by destruction, which could be caused by trauma or intra-abdominal infection by bacteria, parasites, fungi, or a combination of infections.(S. et al., 2014; Michael P. Federle and Siva P. Raman, 2015; Mavilia, Molina and $\mathrm{Wu}, 2016)$ It is broadly divided into pyogenic and amoebic abscess.(Desai et al., 2013) The most common causative organism is polymicrobial.(Khim et al., 2019) Most cases of liver abscess are pyogenic abscesses, which accounts approximately $90 \%$ of liver abscess incidence.(Michael P. Federle and Siva P. Raman, 2015) There are various complications due to liver abscess, and the most common and fatal complication is abscess rupture that might lead to death.(Desai et al., 2013)

\section{Case Report}

A 27 years old male patient came to the hospital with persistent right upper abdominal pain. The pain started one week ago after the patient fell from the stairs, where the right side of the abdomen hit the stairs. The pain progressively worsened in the last three days before hospital admission. The patient described the pain as being "crushed" in that area, but the pain did not radiate to other area. The pain was relieved when the patient took analgesic. The patient felt that his stomach seemed to be getting larger and also complained nausea and vomiting during mealtime for a week before hospital admission, with vomit containing food but no blood. He also complained tea-colored urine for the past week, but no pain during urination. The bowel movements were regular. No complaints of fever, cough or shortness of breath. From physical examination on the abdomen, there was a shifting dullness on percussion and a palpable mass in the right hypochondrium region, radiating to the umbilical area with an approximate size of $10 \times 5 \mathrm{~cm}$ with flat edges.

Blood count analysis showed a hemoglobin level of 11,4 g/dL, an elevated white blood cell count of $14,42 * 10 * 3 / \mathrm{uL}$ and decreased eosinophil level of 0,0-0,1\%. Direct and total bilirubin levels were markedly elevated at 3,14-6,96 and 6,55-6,96*mg/dL. Aspartate aminotransferase (597 U/L) and alanine aminotransferase (466 U/L) levels were also markedly elevated. A coagulation test showed a prolonged clotting time. We did not isolate any microorganism from the blood culture collected in the ER before antibiotic use. Plain chest x-ray returned normal and abdominal x-ray revealed hepatomegaly but no pneumoperitoneum. Abdominal ultrasound revealed a heteroechoic lesion, ill-defined border, irregular margin, with an internal moving echo in the right liver lobe, which on color doppler examination showed minimal perilesional vascularization (figure 1). The patient 
then underwent abdominal CT scan with contrast four days later with the results of rim-enhancing semisolid lesion (23-33 HU) with air density (-925 HU) within it, partially circumscribed, lobulated margins in segment V to VIII of the right liver lobe, which was partially ruptured in the periphery of segment VI-VII of the liver lobe with pneumoperitoneum, ascites, hepatomegaly and right pleural effusion, some lesions appeared as small locules which gathered together to form a "cluster sign" like yellow arrows in the sagittal projection (figure 2).

Five days after the patient was admitted, laparotomy was performed with adhesiolysis, during the operation, the air was sprayed and pus browned $2000 \mathrm{ml}$. A week later, relaparatomy was performed, the peritoneal fluid was clear, pus was absent, hollow organ was intact, cavity abscess in segment 6-7 drained liver. The results of the pus' specimen culture was Escherichia coli with positive-extended-spectrum beta-lactamases (ESBL).
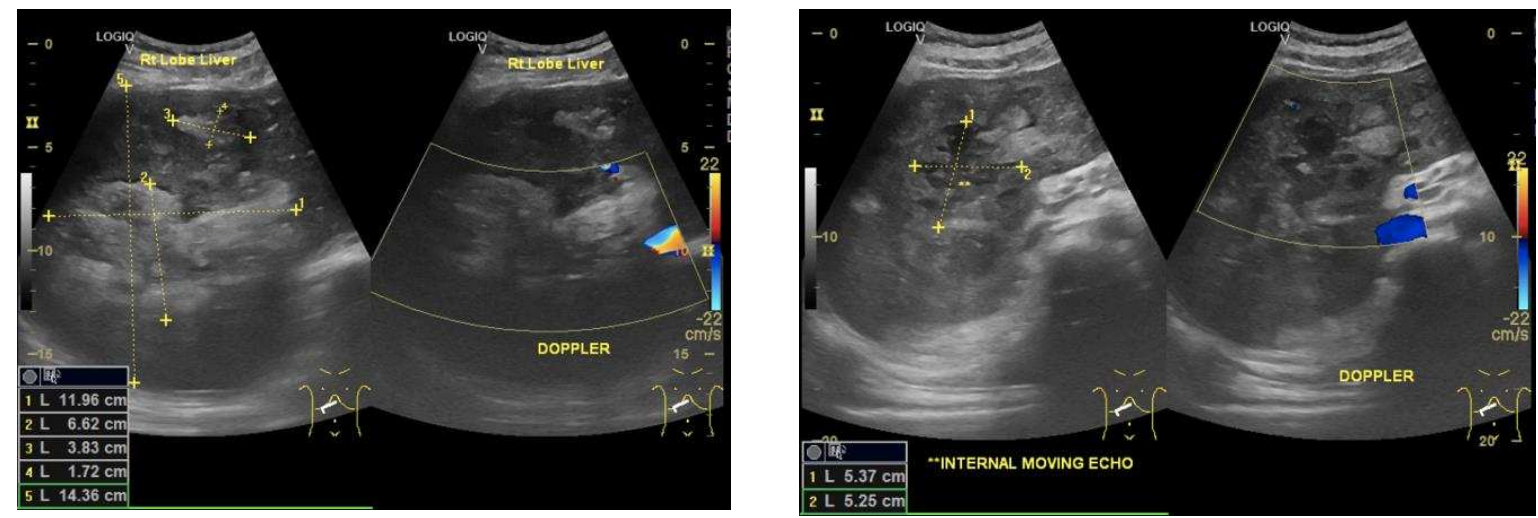

Figure 1. Abdominal Ultrasound, There are heteroechoic lesion was found, ill border, irregular margin, with an internal moving echo (+) in the right lobe of the liver, which on color doppler showed minimal perilesional vascularization.

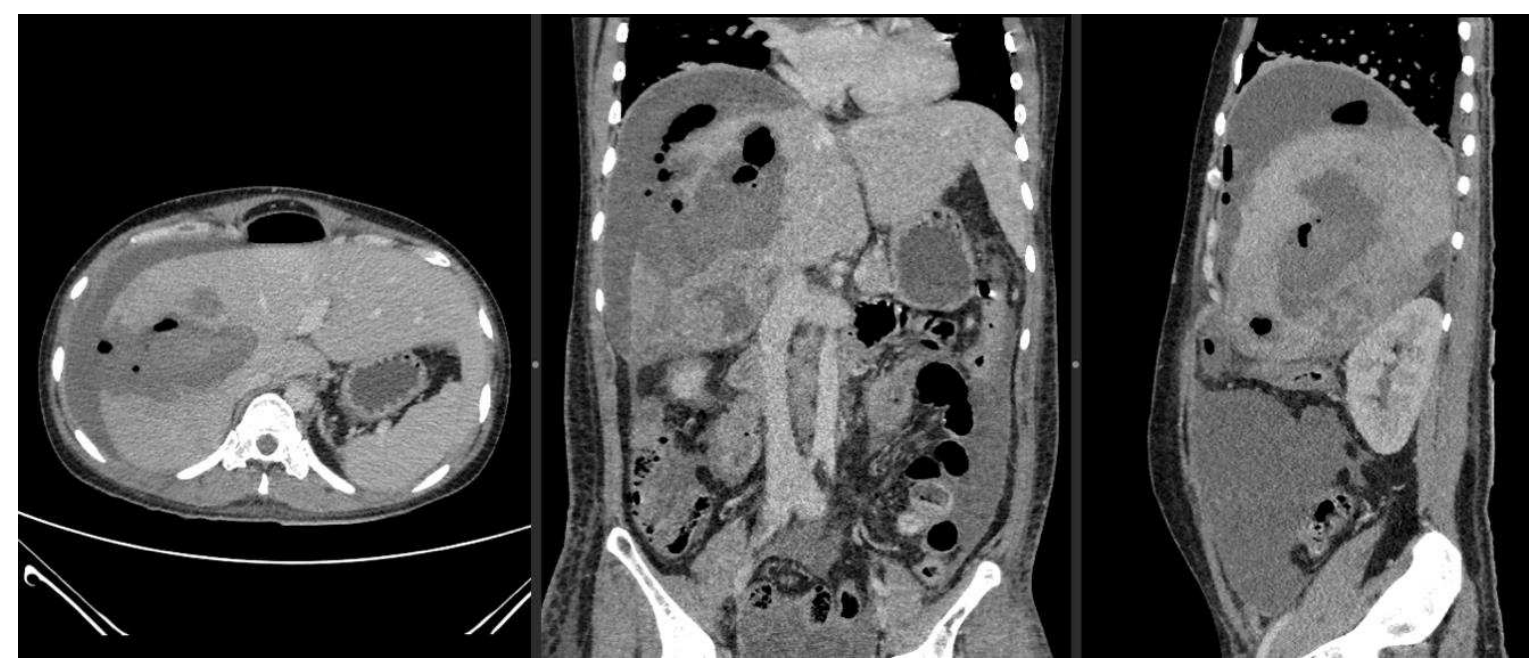




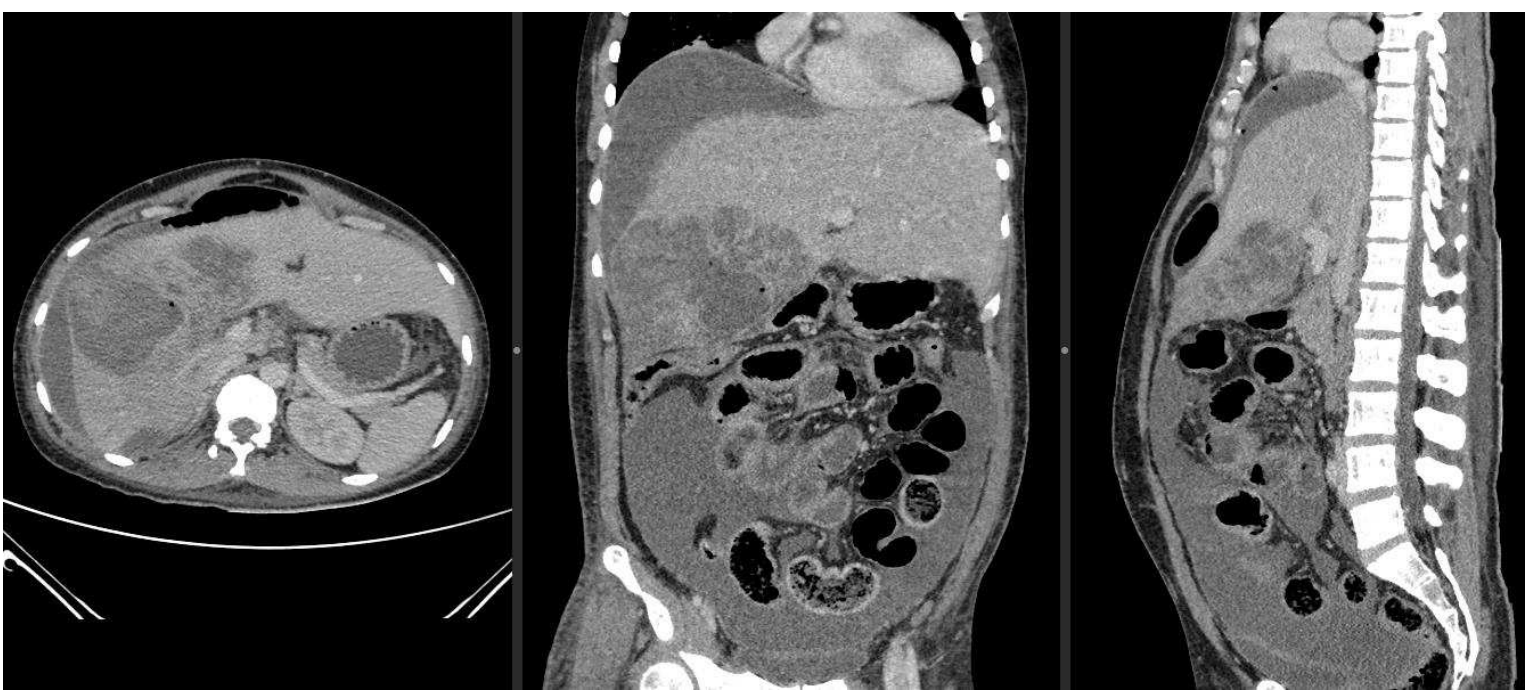

Figure 2. abdominal CT scan with contrast, which showed rim enhancing semisolid lesion (23-33 HU) with air density (-925 HU) within it, partially circumscribed, lobulated margins in segments V to VIII of the right lobe of the liver which partially ruptured in the periphery of segments VI-VII of the liver lobe with pneumoperitoneum, ascites, hepatomegaly and right pleural effusion, some lesions appear as small locules gathered together to form a "cluster sign".

\section{Discussion}

Liver abscess is defined as a collection of encapsulated purulent material in the parenchyma accompanied by destruction of the liver parenchyma, which can be caused by trauma or intra-abdominal infection by bacteria, parasites, fungi, or a combination of infections.(S. et al., 2014; Michael P. Federle and Siva P. Raman, 2015; Mavilia, Molina and Wu, 2016) The abscesses classified into pyogenic and amoebic.(Desai et al., 2013) The cause of liver abscess varies. Half of cases can be from the development of cholangitis. Most cases of liver abscess are pyogenic abscess, about $90 \%$ of the incidence of liver abscess.(Michael P. Federle and Siva P. Raman, 2015) The most common organisms that caused liver abscess include E. Coli, Klebsiella, Streptococcus, Staphylococcus, anaerobic organisms. It is rare to find secondary pneumoperitoneum due to intraperitoneal rupture of a pyogenic liver abscess.(Shiba et al., 2007) Entamoeba hystolytica was the most common cause for anaerobic organisms. Infection in the liver parenchyma can occur due to multifactorial and from several routes, the spread of infection from surrounding organs, blunt trauma or penetrating abdominal trauma, invasive procedures (biliary instrumentation, transcatheter arterial chemoembolization, percutaneous liver biopsy, percutaneous radiofrequency ablation or abdominal surgery), hematogenous spread of gastrointestinal infections via the portal vein, and most commonly systemic bacteremia.(Mavilia, Molina and $\mathrm{Wu}, 2016$ )

Our patient complained of persistent upper right abdominal pain in the past week before hospital admission after the patient fell from the stairs, where the right side of the abdomen hit the stairs. The pain progressively worsened in the last three days before hospital admission and he described the pain as being "crushed" in that area only. Blunt abdominal trauma could result in liver parenchyma ischemia. Abscess formation might be a result from bacterial growth in hematoma or hepatic necrosis area which occurs weeks or months after the initial trauma. Liver parenchyma ischemia is an ideal anaerobic environment for bacterial growth.(Hsieh and Hsu, 2003) Most of the symptoms caused by liver abscess are not specific. However, the most commonly reported symptoms include fever, abdominal pain and hypotension. In a study conducted by Soumik Ghosh et al., 2014 in New Delhi, India, on 200 patients with liver abscess, abdominal pain was the most common symptom (99\%).(Mavilia, Molina and $\mathrm{Wu}, 2016)$ Abscess rupture plays a role in increased morbidity and mortality. Approximately $78 \%$ of cases of amoebiasis peritonitis are due to rupture of an amoebic abscess. It is estimated that an amoebic liver abscess caused around 100,000 deaths.(Khosravani et al., 2017)

Laboratory findings obtained include leukocytosis, eosinophilia, increased erythrocyte sedimentation rate (ESR), HB <10 mg\%, bilirubin $>1 \mathrm{mg} / \mathrm{dl}$, increased alkaline phosphatase, increased serum glutamic oxaloacetic transaminase, increased serum glutamic pyruvic transaminase, abnormal prothrombin time (PTT) and hypoalbuminemia.(Jha et al., 2015) In our patient, showed leukocytosis, eosinophilia, increased liver function, 
abnormal coagulation test and hypoalbuminemia. These results are more directed to the infection process than to malignancy. Radiological imaging plays an vital role in early detection, characterization and management of infectious liver disease.(Bächler et al., 2016) Ultrasonography (US), computed tomography (CT), and magnetic resonance imaging (MRI) have high accuracy in detecting liver infections, even identifying the underlying pathogen.

Blunt abdominal trauma can lead to the risk of liver abscess formation. Abdominal ultrasound examination can detect an abscess in the abdominal organs early. The price is more affordable and can be done immediately, especially in the emergency room. The sensitivity of US and CT scan in detecting pyogenic liver abscess is between $85 \%$ and $97 \%$, respectively. On ultrasound, the picture of the liver abscess will show lesions with irregular edges/midly echogenic wall, septate or fluid level within the lesion, hyperechoic foci with posterior artifacts (gas) and debris with posterior enhancement. In this patient it appeared as a heteroechoic lesion, irregular borders, with internal moving echo $(+)$ within it in the right lobe of the liver. From the ultrasound image, it can lead to an abscess or malignant mass, but the infection can get symptoms of infection and abnormal laboratory results.(Michael P. Federle and Siva P. Raman, 2015; Bächler et al., 2016) On an enhanced CT, a fluid-filled central hypodense area is seen surrounded by an inner hyperintense and outer hypointense area. The lesion is welldemarcated, spherical, multiseptated or cluster of small abscesses and sometimes accompanied by air density $(<20 \%)$. The most common causes of gas-producing bacteria in liver disease are Klebsiella species and Clostridia species. In conditions of abscess rupture, it can cause pneumoperitoneum. In our cases, CT scan showed a rim enhancing semisolid lesion with rupture of the abscess wall on its lateral side with multiple air density filling intraperitoneal perihepatica leading to pneumoperitoneum with ascites. Five days after the patient was admitted, laparotomy was performed, during the operation the air was sprayed and pus browned $2000 \mathrm{ml}$. The results of the pus specimens culture were Escherichia coli with positive extended-spectrum beta-lactamases (ESBL).

In this literature, both clinically, laboratory and radiologically, our case is more suggestive of liver abscess caused by pyogenic bacteria, complicated by abscess rupture. It is evident from the results of the culture of the pus specimen, it shows that the results of Escherichia coli ESBL cultures are obtained. In conclusion, based on clinical symptoms and imaging, PLA is not without any challenges in diagnosis. The key to avoid misdiagnosis of ruptured PLA is a meticulous examination of the clinical and imaging also vigilant monitoring.

\section{References:}

Bächler, P. et al. (2016) 'Multimodality imaging of liver infections: Differential diagnosis and potential pitfalls', Radiographics, 36(4), pp. 1001-1023. doi: 10.1148/rg.2016150196.

Desai, N. et al. (2013) 'Management of Ruptured Liver Abscess: A Study of 54 Cases', International Journal of Science and Research (IJSR) ISSN, 4(1), pp. 2013-2016. Available at: www.ijsr.net.

Hsieh, C. H. and Hsu, Y. P. (2003) 'Early-onset liver abscess after blunt liver trauma: Report of a case', Surgery Today, 33(5), pp. 392-394. doi: $10.1007 / \mathrm{s} 005950300089$.

Jha, A. K. et al. (2015) 'Clinicopathological study and management of liver abscess in a tertiary care center', Journal of Natural Science, Biology and Medicine, 6(1), pp. 71-75. doi: 10.4103/0976-9668.149091.

Khim, G. et al. (2019) 'Liver abscess: Diagnostic and management issues found in the low resource setting', British Medical Bulletin, 132(1), pp. 45-52. doi: 10.1093/bmb/ldz032.

Khosravani, M. et al. (2017) 'The association between conscience understanding and clinical performance among nurses working at education hospital of Arak', Annals of Tropical Medicine and Public Health, 10(6), pp. 1587-1590. doi: 10.4103/ATMPH.ATMPH.

Mavilia, M. G., Molina, M. and Wu, G. Y. (2016) 'The evolving nature of hepatic abscess: A review', Journal of Clinical and Translational Hepatology, 4(2), pp. 158-168. doi: 10.14218/JCTH.2016.00004.

Michael P. Federle, M. and Siva P. Raman, M. (2015) Diagnostic Imaging Gastrointestinal. Third edit. Philadelphia: Elsevier.

S., G. et al. (2014) 'Clinical, laboratory, and management profile in patients of liver abscess from Northern India', Journal of Tropical Medicine, 2014(April 2013), p. no pagination. Available http://www.hindawi.com/journals/jtm/\%5Cnhttp://ovidsp.ovid.com/ovidweb.cgi?T=JS\&PAGE=reference\&D=emed12\&NEWS=N\&A $\mathrm{N}=2014443199$.

Shiba, H. et al. (2007) 'Pneumoperitoneum caused by ruptured gas-containing liver abscess', Journal of Hepato-Biliary-Pancreatic Surgery, 14(2), pp. 210-211. doi: 10.1007/s00534-006-1136-y. 